\title{
A SIMPLE AND FLEXIBLE MOVEMENT CONTROL METHOD FOR A HEXAPOD WALKING ROBOT
}

\author{
YAGUANG ZHU, LIANG ZHANG, WANJIN GUO and ZHENGCANG CHEN \\ The Key Laboratory of Road Construction Technology and Equipment of MOE, Chang'an University \\ Xi'an, 710064, China \\ E-mail: zhuyaguang@chd.edu.cn,zhangliang@chd.edu.cn,guowanjin@chd.edu.cn, \\ chenzhc8905@chd.edu.cn
}

\begin{abstract}
The movements of nature creature such as crawling, walking and running are so flexible and coordinate. Many reseachers turn to Central Pattern Generators (CPG) to make the legged robots be able to have the same behavior ability. In this paper, we combine the model control and bio-inspired contorl for the flexible locomotion control of legged robot to accomplish the omnidirectional movement of the hexapod robot through the Central Pattern based Backward Control method (CPBC). $\sigma$-Hopf oscillator is used as the control unit. Inspired by the compound motion of ants, which is made up of several simple movement forms, the movement trajectory is planned and then body kinematics is used to obtain expected body movement like ants. A hexapod robot named SmartHex is used to carry out the experiment of the omnidirectional movement. The experimental results show that the proposed algorithm can significantly improve the stability and flexibility of the robot, and various movement patterns can be achieved.
\end{abstract}

\section{Introduction}

Inspired by nature creatures, legged robots are often designed to mimic performance of animal for strong terrain passability and movement flexibility. Especially in recent years, some famous legged robots such as Atlas[1], HyQ[2], Cheetah[3] and ANYmal[4] have shown excellent performance. For biped and quadruped robots, the number of legs that can be used to support the torso is very limited in order to achieve mobility, so balance problems and dynamic stability issues are well worth studying. For a hexapod robot, it has more leg numbers and degrees of freedom as well as the reliable support points, so the balance and stability are easily satisfied. But at the same time, the increase of actuators and related structures has also led to more complicated control processes and make it more difficult to achieve reasonable, stable and flexible diversity of motion behaviors. But in fact, there are always answers in the bionic research for all the behaviors of the robot including the coordinated movement of the legs, the trajectory formation of the foot, the adjustment of the body posture, the path selection of approaching target and the behavior after the injury.

In natural hexapods, the locomotion and behavior of ants is very interesting. Movements such as forward, turn, retreat, and sideways are included by all movements. As shown in Figure 1, in the process of approaching the target point from the starting point, there is almost no straight line. And even if a straight line exists, it is often with sideways, moving obliquely to the target position. The behavior is completely different from most robots. At present, the behavior planning of mobile robots simplifies the robot into a mass point, and performs behavior planning such as straight lines, arcs, and splines. Of course, through continuous improvement and optimization, the refinement path can completely achieve a random curve similar to the ant moving path. However, the physical behavior of ants is not based on the mass point, but through the control of the center of mass, the trunk orientation and the legs coordination to complete the flexible overall movement. 


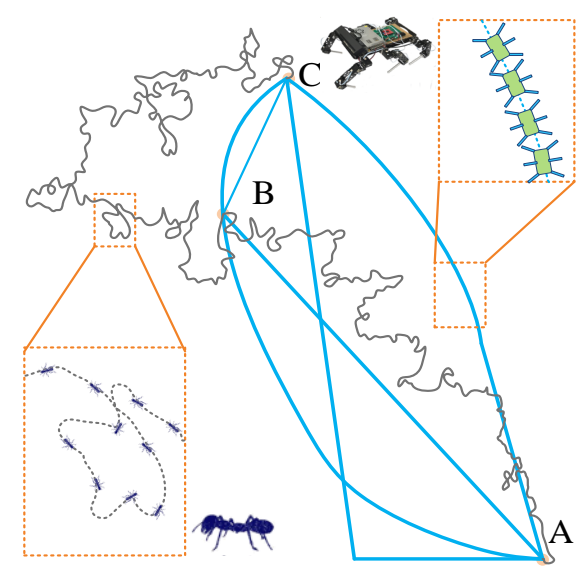

Figure 1. Compound motion of an ant and movement of a hexapod robot.

The study of the adaptive and flexible movement is an important in the bio-inspired legged robot. Some researchers are inclined to use the model control methods to achieve the various forms of movement. The virtual model control method is applied to the BigDog [5]. The adaptive control belonging to behavior-based control method is used to adapt to the unstructured environment [6-8]. The Biological control methods are also hot research topics at present. The AMOS [9] is controlled by a neurocontroller that generates the basic locomotion and controls the sensor-driven behavior of the robot. An adaptive control system of coupled nonlinear oscillators achieved the omnidirectional movement $[10,11]$. And a heading direction controller based on CPG proposed by Vitor Mators et al. [12] is able to adapt to sensory-motor visual feedback, and online adjust its trajectory according to visual information that modifies the control parameters. Actually, it is not easy to control the hexapod robot as flexible as ant. From the perspective of bionic control, although CPG can easily implement gait switching or behavioral transition, this method is not accurate for the position control of the legs, which makes foot trajectory unsmooth and unregular. In this case, the torso of robot may not be able to keep stable or even balance because of the different speed and uncoordinate positon of each leg. The reason is that conventional bionic control lacks precise constraints on leg behavior and torso behavior, which contributes to an unsmooth and unsatisfactory final movement. Therefore, this paper proposes a smiple control method based on Central Pattern based Backward Control (CPBC), which enables the robot to achieve leg coordination under the rythm of CPG signals, and achieve precise control of the torso and legs. The combined motion of the robot is obtained through the analysis of the motion process of ant. Therefore, the advantages of both model control and bio-inspired control are taken into consideration.

\section{Materials and Methods}

\subsection{Control architecture}

The hexapod robot platform, SmartHex [13] used for experiment is designed in the principle of energy-efficiency and bionic idea. Its omnidirectional movement is driven by CPG. The whole control frame includes the module of $\sigma$-Hopf harmonic oscillator, gait generator, omnidirectional movement path generator, foot trajectory generator and inverse kinematics solver, as is shown in the Figure 2. The oscillator generates signal $x$ and $y$, which have same frequency and a half-cycle phase difference. Through the coefficient of phase shift $\zeta$, the signal $x$ turns to be the six controlling signals having certain phase difference between 
them, which are used to solve the equation of foot tip trajectory. And the planning process depends on the signal $y$. When $y>0$, the signal $x$ is on the rise, which is used to drive the equation of swing phase trajectory of the foot. When $y<0$, the signal $x$ is on the downside, which is used to solve the equation of supporting phase trajectory of the foot. The polynomial curve is used for the foot trajectory and the fitting parameters are produced by the omnidirectional movement path generator through the mapping relationships. Finally, the generated foot trajectory is obtained by inverse kinematics solver to obtain the joint driving signals.

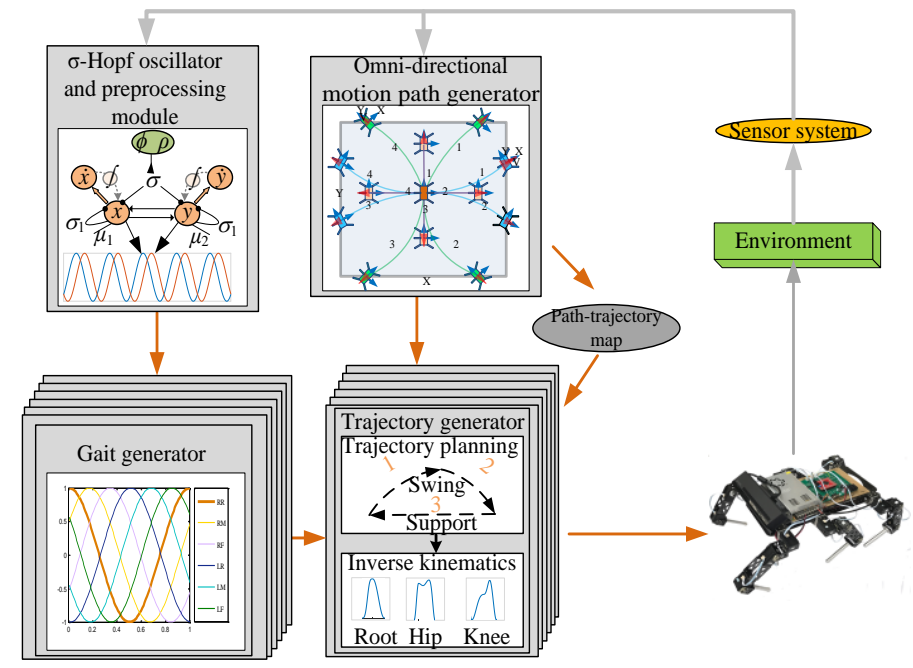

Figure 2. Control architecture of the Combined Control Method

\section{2. -Hopf oscillator}

-Hopf harmonic oscillator [14] is a module of the central pattern generator (CPG). The output frequency and amplitude are easy to adjust. The stable amplitude is limited from -1 to 1 , forming a steady limit cycle. At same time, the oscillator generats stable periodic signals $x$ and $y$, which start from random values, and turn to be the stable values in a short time and then oscillate at a adjustable phase difference. Finally, the decoupling operation is applied to the $\sigma$ Hopf harmonic oscillator, which makes duty ratio and gait uncorrelated, shown in the Figure 2. The mathematical expression of the oscillator is shown in the Eq. (1) and (2):

$$
\begin{aligned}
& \left\{\begin{array}{l}
\dot{x}=\alpha\left(\mu-r^{2}\right)\left(x-\mu_{1}\right)-\sigma\left(y-\mu_{2}\right) \\
\dot{y}=\alpha\left(\mu-r^{2}\right)\left(y-\mu_{2}\right)-\sigma\left(x-\mu_{1}\right) \\
r^{2}=\left(x-\mu_{1}\right)^{2}+\left(y-\mu_{2}\right)^{2} \\
\sigma=\pi /\left(\rho \cdot\left(e^{-a y}+1\right) \cdot \varphi\right)+\pi /\left((1-\rho) \cdot\left(e^{a y}+1\right) \cdot \varphi\right)
\end{array},\right. \\
& x_{i}(t)=\left\{\begin{array}{ll}
x(t-(1-i) \cdot(1-\zeta) \cdot \phi) & (i=1,2,3) \\
x(t+(k+4-i) \cdot(1-\zeta) \cdot \phi) & (i=4,5,6)
\end{array} .\right.
\end{aligned}
$$

where $\mathrm{x}$ and $\mathrm{y}$ are the state variables; $\mathrm{r}$ is the radius of the limit cycle; $\sqrt{\mu}$ is the amplitude of the oscillations, $\alpha$ is the positive constant that controls the speed of convergence of the limitcycle; $\mu_{1}$ and $\mu_{2}$ are external feedback; $\rho$ is the duty factor; $\phi$ is the output period. $\zeta$ is the phase shift coefficient and determines the phase difference of the output signals transmitted to the six legs for the different gaits. 


\subsection{Ant-inspired movement control algorithm}

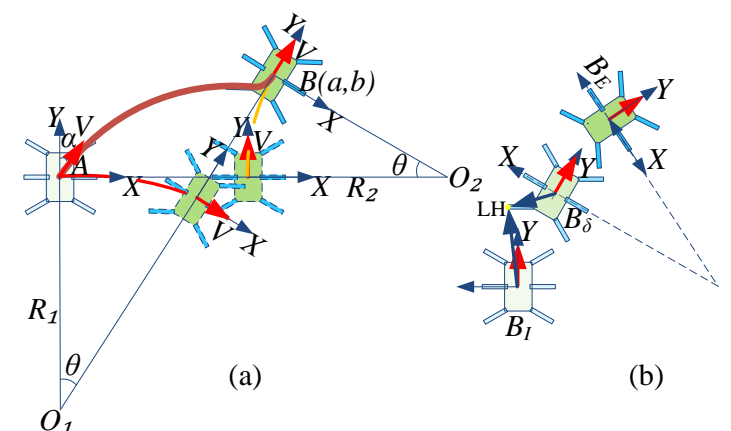

Figure 3. (a) Omnidirectional movement. (b) COG Trajectory of turning process;

In this paper, the omnidirectional movement of the robot is decomposed into two submotions: a turning motion along the direction of the forward and a turning motion along the direction of the side of the body. Assuming that the robot moves from position $A(0,0)$ to position $B(a, b)$ during a gait cycle, the axes of center of gravity (COG) at location $A$ and location $B$ are respectively extended to intersect at point $O_{1}$ and point $O_{2}$, which are the centers of the two turning movements in the Figure 3(a). Obviously, the movement of the robot can be seen as the combination of two turning movements. After a gait cycle, the value of heading angle of the robot turns to be $\theta$. Now, the reference frame $X O Y$ is built in the initial position $A$ of the robot, which coincides with the COG coordinate system. Based on the geometrical relationship, the value of turning radius $R_{1}, R_{2}$ can be deducted to:

$$
\left\{\begin{array}{l}
R_{1}=a / \tan \theta-b \\
R_{2}=b / \sin \theta
\end{array} .\right.
$$

Then, the velocity $v$ is introduced to control the magnitude and the direction of velocity of the robot. And, the angle between velocity vector and the $Y$ axis in reference frame is defined as variable $\alpha$. Finally, $a, b, v, \alpha$ are used as the parameters of constraint conditions to compute the coefficients of cubic polynomial so as to fit the cubic polynomial curve, which is used as the trajectory of COG. Equation for trajectory of COG is

$$
B_{C O G}(x)=\left(\begin{array}{l}
X_{C O G} \\
Y_{C O G}
\end{array}\right)=\left(\begin{array}{l}
a_{0 x}+a_{1 x} x+a_{2 x} x^{2}+a_{3 x} x^{3} \\
a_{0 y}+a_{1 y} x+a_{2 y} x^{2}+a_{3 y} x^{3}
\end{array}\right) .
$$

Constraint conditions are

$$
B_{C O G}(-1)=\left(\begin{array}{l}
0 \\
0
\end{array}\right), B_{C O G}(1)=\left(\begin{array}{l}
a \\
b
\end{array}\right), \dot{B}_{C O G}(-1)=\left(\begin{array}{l}
-v \sin \alpha \\
-v \cos \alpha
\end{array}\right), \dot{B}_{C O G}(1)=\left(\begin{array}{l}
-v \sin (\alpha+\theta) \\
-v \cos (\alpha+\theta)
\end{array}\right) .
$$

The constraint conditions are substituted into Eq. (4) to compute the coefficients of cubic polynomials. Then the trajectory of COG can be obtained. In Eq. (4), $x$ is not the time, but the output signal of the CPG module. $a_{n x}$ and $a_{n y}$ are the coefficients of the cubic polynomial. $X_{C O G}$ and $Y_{C O G}$ are the coordinate point of COG. According to Eq. (2) and (3), the trajectory of the robot between position $A$ and position $B$ is completely controlled by $a, b, \theta, v, \alpha$ in a gait cycle.

Taking the front-right turning motion with radius $R$ as an example in the Figure 3(b), if the robot needs to achieve turning motion, the coordinate value of point $B$ should satisfy this equation after a gait cycle: 


$$
\left\{\begin{array}{l}
a=R-R \cos \theta \\
b=R \sin \theta
\end{array}\right.
$$

Substituting Eq. (6) into Eq. (3) to obtain:

$$
\left\{\begin{array}{l}
R_{1}=(R \cos \theta-R) / \sin \theta \\
R_{2}=R
\end{array} .\right.
$$

In summary, the more special movement forms can be seen in the Figure 4 and the parameters of these movement forms can be seen in table 1 .

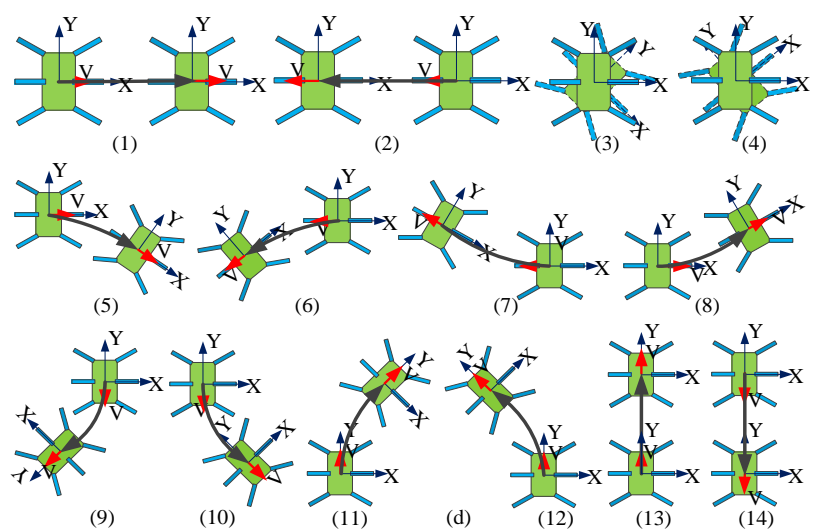

Figure 4. Special movement forms 1. translational movement to right. 2. translational movement to left. 3. spot turning to right. 4. spot turning to left. 5. right-backward movement with radius $R_{l}$. 6. left-backward movement with radius $R_{l}$. 7. left-forward movement with radius $R_{l}$. 8. right-forward movement with radius $R_{2}$. 9. leftbackward movement with radius $R_{2}$. 10. right-backward movement with radius $R_{2}$. 11. right-forward movement with radius $R_{2}$. 12.left-forward movement with radius $R_{2}$. 13. forward movement. 14. backward movement.

Table 1 Parameters of the special movement forms

\begin{tabular}{ccccc}
\hline & $R_{I}$ & $R_{2}$ & $\alpha / \mathrm{rad}$ & $\theta / \mathrm{rad}$ \\
\hline 1 & $S / \tan \theta$ & 0 & $\pi / 2$ & $>0$ \\
2 & $S / \tan \theta$ & 0 & $\pi / 2$ & $<0$ \\
3 & 0 & 0 & 0 & $>0$ \\
4 & 0 & 0 & 0 & $<0$ \\
5 & $R$ & $\left(R_{1} \cos \theta-R_{1}\right) / \sin \theta$ & $\pi / 2$ & $>0$ \\
6 & $R$ & $\left(R_{1} \cos \theta-R_{1}\right) / \sin \theta$ & $\pi / 2$ & $<0$ \\
7 & $-R$ & $\left(R_{1} \cos \theta-R_{1}\right) / \sin \theta$ & $\pi / 2$ & $>0$ \\
8 & $-R$ & $\left(R_{1} \cos \theta-R_{1}\right) / \sin \theta$ & $\pi / 2$ & $<0$ \\
9 & $\left(R_{2} \cos \theta-R_{2}\right) / \sin \theta$ & $-R$ & $-\pi$ & $>0$ \\
10 & $\left(R_{2} \cos \theta-R_{2}\right) / \sin \theta$ & $R$ & $-\pi$ & $<0$ \\
11 & $\left(R_{2} \cos \theta-R_{2}\right) / \sin \theta$ & $R$ & 0 & $>0$ \\
12 & $\left(R_{2} \cos \theta-R_{2}\right) / \sin \theta$ & $-R$ & 0 & $<0$ \\
13 & $-R_{2} \sin \theta$ & $S / \tan \theta$ & 0 & $<0$ \\
14 & $-R_{2} \sin \theta$ & $S / \tan \theta$ & $-\pi$ & $>0$ \\
\hline
\end{tabular}

\subsection{Foot tip trajectory planning}

The movement of the multi-legged robot is realized through the alternating movements of the legs in a certain time order, and the motion of each leg can be seen as the alternating movements between the support phase and swing phase. The support phase is used for supporting the robot body and achieving the movement of the robot through interaction of the joints. When the foot tip of the robots is at the final moment of the support phase, the foot tip lifts and then falls to the starting point of next swing phase. When a leg of the robot is in the support phase, the foot tip of the leg, which is moving relative to the COG coordinate system of 
the robot body, is stationary relative to the ground. In this paper, the geodetic coordinate system is established in the center of turning trajectory, which is used as the reference coordinate system. When the robot starts turning, the COG coordinate system of the body will move along the arc with the radius $R$. The negative direction of $x$ axis always points to the center of turning trajectory. In the Figure 3(b), the COG coordinate system of the body changes from $B_{I}$ to $B_{E}$ after a gait cycle, and the position of the foot tip can be expressed as ${ }^{B_{I}} P_{A_{i}}$ under the COG coordinate system $B_{I}$. When the robot body moves to a random position $B_{\delta}$ in a gait cycle, the rotation operator and translational operator from coordinate system $B_{I}$ to the coordinate system $B_{\delta}$ :

$$
{ }_{B_{I}}^{B_{\delta}} R=\left(\begin{array}{ccc}
\cos \delta & -\sin \delta & 0 \\
\sin \delta & \cos \delta & 0 \\
0 & 0 & 1
\end{array}\right) \cdot{ }^{B_{\delta}} P_{B_{I} O R G}=\left(\begin{array}{l}
-X_{C O G} \cos \theta+Y_{C O G} \sin \theta \\
-X_{C O G} \sin \theta-Y_{C O G} \cos \theta \\
0
\end{array}\right) .
$$

According to the transformation method of space coordinate system, the coordinate of foot tip in coordinate system $B_{I}$ :

$$
{ }^{B_{\delta}} P_{A_{i}}={ }_{B_{I}}^{B_{\delta}} R^{B_{I}} P_{A_{i}}+{ }^{B_{\delta}} P_{B_{I} O R G} .
$$

Thus, Eq. (10) can be rewriten to obtain the foot trajectory of supporting phase:

$$
{ }^{B_{\delta}} P_{A_{i}}=\left(\begin{array}{l}
{ }^{B_{\delta}} X_{A_{i}} \\
{ }^{B_{\delta}} Y_{A_{i}} \\
{ }^{B_{\delta}} Z_{A_{i}}
\end{array}\right)=\left(\begin{array}{l}
{ }^{B_{I}} X_{A_{i}} \cos \delta-{ }^{B_{I}} X_{A_{i}} \sin \delta-X_{C O G} \cos \delta+Y_{C O G} \sin \delta \\
{ }^{B_{I}} X_{A_{i}} \sin \delta+{ }^{B_{I}} Y_{A_{i}} \cos \delta-X_{C O G} \sin \delta-Y_{C O G} \cos \delta \\
{ }^{B_{I}} Z_{A_{i}}
\end{array}\right) .
$$

in which, $\delta=(-\theta \cdot \rho) \cdot x / 2+\theta \cdot \rho / 2 . \rho$ is the duty factor. The swing phase trajectory is used for connecting the starting and ending points of the supporting phase trajectory. When foot tip touching and leaving the ground, the swing phase ensures that there is no impact between foottip and ground. Then, take the derivative of $x$ (output signal of $\sigma$-Hopf oscillator) in Eq. (9) to compute the velocity of swing phase:

$$
\begin{gathered}
V_{A_{i}}=\left(\begin{array}{l}
v_{x i}(x) \\
v_{y_{i}}(x) \\
v_{z i}(x)
\end{array}\right)=\left(\begin{array}{l}
(\theta \cdot \beta / 2) \cdot M-X_{\text {diff }} \cos \delta+Y_{\text {diff }} \sin \delta \\
(\theta \cdot \beta / 2) \cdot N-X_{\text {diff }} \sin \delta-Y_{\text {diff }} \cos \delta \\
0
\end{array}\right) . \\
M=\left(-{ }^{B_{I}} X_{A_{i}} \sin \delta+{ }^{B_{I}} Y_{A_{i}} \cos \delta+X_{C O G} \sin \delta+Y_{C O G} \cos \delta\right) . \\
N=\left({ }^{B_{I}} X_{A_{i}} \sin \delta-{ }^{B_{I}} Y_{A_{i}} \cos \delta-X_{C O G} \sin \delta+Y_{C O G} \cos \delta\right) .
\end{gathered}
$$

in which, $X_{\text {diff }}$ and $Y_{\text {diff }}$ are the velocity components of COG. $V_{A i},{ }^{B_{\delta}} P_{A_{i}}$ are used as the parameters of constraint conditions to compute the coefficients of cubic polynomial curve, which use as the swing phase trajectory. Equation for swing phase trajectory is

$$
F_{t i p}(x)=\left(\begin{array}{l}
X_{t i p} \\
Y_{t i p} \\
Z_{t i p}
\end{array}\right)=\left(\begin{array}{l}
a_{0 x}+a_{1 x} x+a_{2 x} x^{2}+a_{3 x} x^{3}+a_{4 x} x^{4} \\
a_{0 y}+a_{1 y} x+a_{2 y} x^{2}+a_{3 y} x^{3}+a_{4 y} x^{4} \\
a_{0 z}+a_{1 z} x+a_{2 z} x^{2}+a_{3 z} x^{3}+a_{4 z} x^{4}
\end{array}\right)
$$

Several constraint conditions, e.g. positions and velocities are used to compute the coefficients of quartic polynomials. Then the swing phase trajectory can be obtained. In the ideal condition, 
the various complex motion forms can be achieved well through ant-inspired movement control algorithm. In case of existing rough terrain, slippage of legs and missing foot holds, body posture will be modified by body trajectory generator to adapt these disturbances.

\section{Experiment and result}

To verify the generality and flexibility of the algorithm in this research, an experiment is carried out using the hexapod robot platform and ant experimental platform. The ant experiment including various movement forms which consist of forward movement, forwardleft movement, forward-right movement and forward-left movement in the Figure 5(a). And then the hexapod robot experiment is carried out to imitate the movement forms of ant, as is shown in the Figure 5(b). The outstanding feature of the omnidirectional movement control algorithm is that it can achieve multiple stable and flexible movement forms like the ant.

The corresponding attitude data and current data are shown in the Figure 5(c). The range of the pitch angle and roll angle are all less than $\pm 0.01 \mathrm{rad}$. There is no obvious fluctuation during each transition. According to the curves of the yaw angle, from $0-3 \mathrm{~s}$, the yaw angle is approximately horizontal and the robot moves straight; From 3-13s, the yaw angle is a slash and the robot makes a forward-right movement with radius $R_{2}$; From 13-16.5s, the yaw angle continues decrease and the robot makes a spot turning; From 16.5-20.5s, the yaw angle remains unchanged and the robot moves straight; From 20.5-25s, the yaw angle has a increase and the robot makes a forward-left movement with radius $R_{2}$; From $25-28.5 \mathrm{~s}$, the yaw angle decreases and the robot makes a forward-right movement with radius. What we should notice is that transition of the different movement costs $0.5 \mathrm{~s}$. This fits perfectly with the preset motion parameters. Therefore, the omnidirectional movement algorithm could achieve a fast transition in different motion states and has good stability and flexibility. The period of the current curve is $1 \mathrm{~s}$, and each period has a peak value that is equal to the gait period.
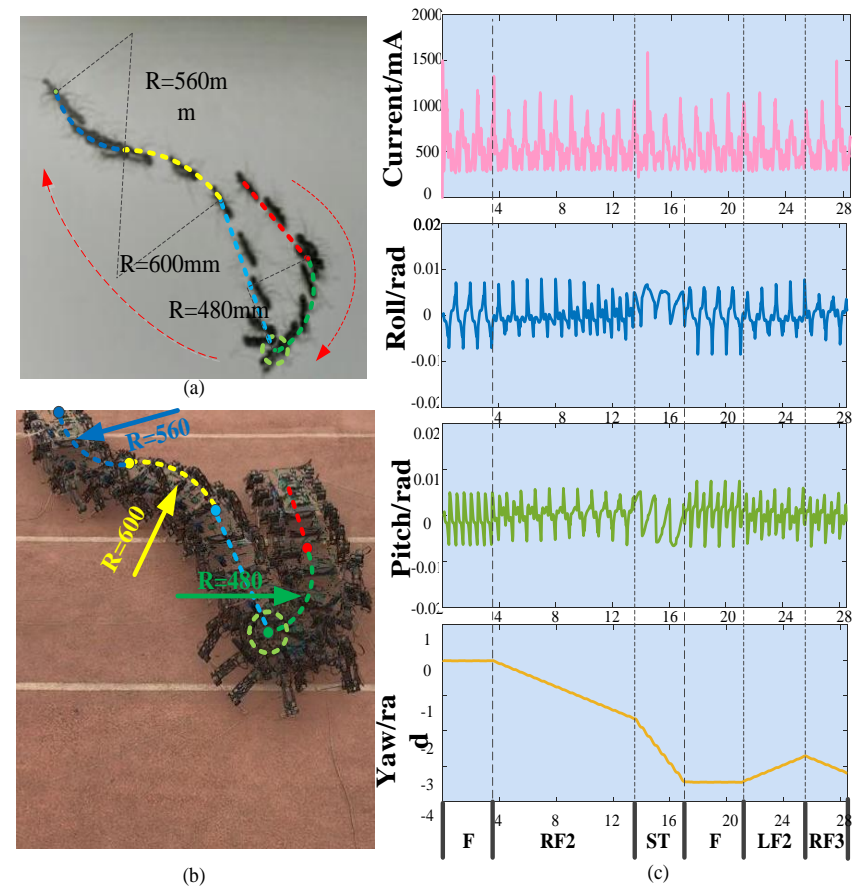

Figure 5. (a) Movement of an ant. (b) Movement of hexapod robot. (c) Attitude angles and current value (F: forward movement. RF2: right-forward movement with radius of $\mathrm{R}=480 \mathrm{~mm}$. STL: spot turning to left movement. LF2: left-forward movement with $\mathrm{R}=600 \mathrm{~mm}$. RF3: right-forward movement with $\mathrm{R}=560 \mathrm{~mm}$.). 


\section{Conclusion}

In order to make the multi-legged robot have the same movement ability as ants, this paper proposes a simple and effective behavior control strategy to combine the model control and bionic control methods to obtain their respective advantages in behavior control. By analyzing the behaviors and habits of ants during the movement process, the conventional robot motion behavior is decomposed and the precise behavior control method is formed by synthesizing the torso trajectory and the leg trajectory, and the coordinated movement between the legs is completed by decoupled CPG module. By making the SmartHex robot imitate behavioral movement of ants, the possibility and effectiveness of the method are verified. These observations illustrate three advantages of this research over existing work: (1) With the decoupled CPG module, the method can quickly complete smooth switching of different gait patterns and achieve fast motion frequency conversion. (2) Through the trajectory planning of torso and legs, a variety of motion modes can be realized, showing great behavioral control ability. (3) The method has smooth transition between motion modes and stable locomotion, which gives the robot extremely flexible behavioral ability. In the future, we will further enhance the ability of robot to have stable body control, making it not only flexible, but also effective in environmental adaptability and passability.

\section{Acknowledgments}

This research was funded by the National Natural Science Foundation of China (No. 51605039), the Thirteenth Five-Year Plan Equipment Pre-research Field Fund (No. 61403120407), the China Postdoctoral Science Foundation (No. 2018T111005 and 2016M592728), Fundamental Research Funds for the Central Universities, CHD (No. $300102259308,300102258203$ and 300102259401$)$.

\section{References}

1. https://www.bostondynamics.com

2. G. Ruben, P. Diego, B Jonas, IEEE Robotics and Automation Letters. 3, 2291 (2018).

3. W. P. Hae, P. Sangin, K. Sangbae, IEEE International Conference on Robotics and Automation. 5163 (2015).

4. H. Jemin., L. Joonho, D. Alexey and B. Dario, T. Vassilios, K. Vladlen, H. Marco. SCIENCE ROBOTICS. 5872 (2019).

5. M. Raibert, K. Blankespoor, G. Nelson, R. Playter, and the BigDog Team, In Proc. of IFAC world congress, 41, 108222008.

6. R. A. Brooks. Neural Computation, 1, 253 (1989).

7. H. Kimura, Y. Fukuoka, Adaptive Motion of Animals and Machines (2000).

8. V. R. Kumar and K. J. Waldron, Joumal of Robotic Systems. 6, 49 (2010).

9. P. Manoonpong, F. Pasemann and H. Roth, Int. J. Rob. Res. 26, 301 (2007).

10. J. Ayers and J. Crisman, Biological Neural Networks in Invertebrate Neuroethology and Robotics, (1992).

11. X. Li, W. Wang, B. Li, Y. J. Wang and Y. P. Yang, Proceedings of the 2009 IEEE, International Conference on Robotics and Biomimetics, 15, 2068, (2009).

12. V. Matos and C. P. Santos, IEEE/RSJ International Conference on Intelligent Robots and Systems, 3392 (2010).

13. Y. G. Zhu, T. Guo, Q. Liu, Q. W. Zhu, X. M. Zhao, B. Jin, Sensors. 17, (2017).

14. Y. G. Zhu, Y. S. Wu, Q. Liu, T. Guo, R. Qin, and J. Z. Hui, Robotics and Autonomous Systems. 106, 165 (2018). 